\title{
Thank You to Our Reviewers
}

We are indebted to the expert referees who have volunteered their time to review submissions for The Canadian Journal of Neurological Sciences in 2020. The editors, authors and readers are immensely grateful for their thoughtfulness and expertise that have served our journal well.

\author{
Abraham, Alon \\ Abrahao, A. \\ Adatia, Sweta \\ Adesina, Ore-ofe \\ Ajani, Olufemi \\ Alcock, Susan \\ Alshimemeri, Sohaila \\ Al-Shorafat, Duha \\ Altman, Robert \\ Amoozegar, Farnaz \\ Anderson, Dustin \\ Andrade, Danielle \\ Ang, Lee Cyn \\ Angel, Michael \\ Anlar, Banu \\ Appel-Cresswell, Silke \\ Appireddy, Ramana \\ Aquino, Camila \\ Armstrong, Melissa \\ Atkinson, Jeffrey \\ $\mathrm{Au}$, Karolyn \\ Avila, Aresnio \\ Ba, Fang \\ Bagnas, Marjorie Anne \\ Baharnoori, Moogeh \\ Baker, Steven \\ Bayram, Ece \\ Beal, Deryk \\ Becker, Werner \\ Beecher, Grayson \\ Ben-Israel, David \\ Benito-León, Julián \\ Berbrayer, David \\ Bergamaschi, Eduardo \\ Bergeron, David \\ Bergeron, Glen \\ Bharatha, Aditya \\ Bhat, Rakesh \\ Blacquiere, Dylan \\ Bocti, Christian \\ Borlot, Felippe \\ Bouchard, Manon \\ Boulias, Chris \\ Boulton, Mel \\ Bove, Francesco \\ Bowden, Gregory \\ Brais, Bernard \\ Breiner, Ari
}

BresBullrich, Maria

Briemberg, Hannah

Bril, Vera

Bruno, Veronica

Budhram, Adrian

Bui, Esther

Burneo, Jorge

Bursztyn, Lulu

Burton, Jodie

Byworth, Miles

Camicioli, Richard

Caplan, Louis

Carlen, Peter

Carruthers, Robert

Carter, Melissa

Catanese, Luciana

Cavallieri, Francesco

Chakrabarti, Rahul

Chalil, Alan

Chalk, Colin

Chan, David

Chan, Ming

Chan, Tommy Lik Hang

Chapman, Kristine

Chaudhary, Neeraj

Chen, Kai-Hsiang Stanley

Chen, Tychicus

Choudhury, Nabajit

Chow, Michael

Chrenek, Ryan

Chrestian, Nicholas

Christie, Suzanne

Chu, Joseph

Climans, Seth

Cloutier, Martin

Connolly, Barbara

Cooper, Paul

Cortes, Maria

Cossetto, Tyler

Costello, Fiona

Cote-Mantha, Evelyne

Couillard, Philippe

Danyluk, Hayden

Daoust, Raoul

Darsaut, Tim

Das, Sumit

Datta, Anita

Day, Gregory de Guise, Elaine

De Roxas, Ranhel

de Villers-Sidani, Etienne

De Vivo, Darryl

De Vleesschouwer, Steven

Dea, Nicolas

Debicki, Derek

Deis, Nathan

Del Bigio, Marc

del Campo, Martin

Di Maio, Salvatore

Dilli, Esma

Diringer, Michael

Dmytriw, Adam

Donaldson, Laura

Donner, Elizabeth

Dorotan, Maria Kristina

Dorovini-Zis, Katerina

Dowlatshahi, Dar

Dukelow, Sean

Dunham, Christopher

Dupré, Nicolas

Duquette, Pierre

Eagles, Matthew

Edwards, Jodi

Ehrensperger, Eric

Eisenstat, David

Elleker, M. George

Fallah, Aria

Fantaneanu, Tadeu

Fasano, Alfonso

Findlay, J.

Fornazzari, Luis

Frauscher, Birgit

Freedman, Mark

Freeman, WD

Freund, Paul

Fridman, Sebastian

Fritzler, Marvin

Ganesan, Rishi

Ganos, Christos

Gao, Andrew

Garcia-Estrada, Everardo

Gasca-Salas, Carmen

Genge, Angela

Ghosh, Soumya

Gioia, Laura

Girgis, Fady
Go, Tina

Gofton, Teneille

Gold, David

Goodridge, Alan

Gospe, Sydney

Gotman, Jean

Gould, Peter

Green, Robin

Hader, Walter

Hahn, Christopher

Haji, Faizal

Hakim, Antoine

Hall, Devin

Halmagyi, Michael

Hamilton, Mark

Hammond, R.

Hanganu, Alexandru

Hanley, Daniel

Hawryluk, Gregory

Hébert, Julien

Henri-Bhargava, Alexandre

Hill, Michael

Hobbs, Hailey

Hoffman, Mark

Hogan, Matthew

Honey, C. Michael

Honey, Christopher

Hunt, Cindy

Huot, Philippe

Hussain, Mohammed

Ibrahim, George

Imran, Jivraj

Ing, Edsel

Iorio-Morin, Christian

Ito, Shinya

Jackson, Alan

Jain, Puneet

Jalini, Shirin

Jansen, Gerard

Jarvis, Carl

Jeerakathil, Thomas

Jenkins, Mary

Jichici, Draga

Jog, Mandar

Johnston, Janine

Josephson, Colin

Joshi, Charuta

Joshi, Sucheta 
Jukes, Alitair

Jurkiewicz, Michael

Kaderali, Zul

Kameda-Smith, Michelle

Kaminska, Marta

Karnik, Vikram

Kasper, Ekkehard

Kassardjian, Charles

Kate, Mahesh

Katzberg, Hans

Kawamura, Anne

Kazina, Colin

Kesarwani, Rohit

Khaw, Alexander

Khosravani, Houman

Kimiskidis, Vasilios

Kirk, Andrew

Kirton, Adam

Kiwan, Ruba

Klein, Eran

Klepper, Joerg

Koh, Yeow Hoay

Krishnan, Pradeep

Krysko, Kristen

Kuhlman, Greg

Kukkle, Prashanth

Kulasingam, Vathany

Kumar, Hrishikesh

Lafontaine, Anne-Louise

Laforce, Robert $\mathrm{Jr}$

Lagman-Bartolome, Ana-Marissa

Lam, Victor

Lattanzi, S

Leal, Paulo

Lee, Liesly

Lehman, Vance

Leroux, Elizabeth

Letourneau-Guillon, Laurent

Levin, Myron

Levy, Ariel

Lewis, Evan

Liang, Shuli

Lidstone, Sarah

Lim, Gerald

Lim-Fat, Mary Jane

Lin, Amy

Lioutas, Vasileios

Lippmann, Kristina

Liu, Grant

Lizarraga, Karlo

Lo, Alexander

Lo, Benjamin

Longoni, Giulia

Lownie, Stephen

Lozano, Andres

Lu, Jian-Qiang

Lynch, Peter

MacDougall, Keith

Magnusson, Linda
Maguire, John

Mailo, Janette

Mandel, Jacob

Mandzia, Jennifer

Mansfield, Avril

Mansouri, Behzad

Marcoux, Judith

Margolin, Edward

Marin, Samatha

Mark, Victor

Marras, Connie

Martin, Wayne

Martino, Davide

Mason, Mark

Massie, Rami

Mathieu, David

Maurice, Catherine

McCredie, Victoria

McDonald, Patrick

McNeely, P. Daniel

Medhi, Gorky

Megyesi, Joseph

Mehta, Vivek

Mercimek-Andrews, Saadet

Mesterman, Ronit

Mestre, Tiago

Metz, Luanne

Mezei, Michelle

Micieli, Jonathan

Midha, Rajiv

Miller, Tom

Mirsatarri, Seyed

Mizzi, Trent

Modrego, Pedro

Mohamed, Ismail

Mohammad, Mohammad

Monges, Soledad

Montanera, Walter

Moore, Fraser

Morrow, Sarah

Muller-Felber, Wolfgang

Munhoz, Renato

Munoz, David

Muthusami, Prakash

Naeem, Abdul

Nair, Jay Kumar

Naldi, Andrea

Nascimento, Fabio

Nayar, Suresh

Nelson, Kate

Netravathi, M

Neva, Jason

$\mathrm{Ng}$, Marcus

Nguyen, Dang

Nibali, Marco Conti

Nichol, Alan

Nicolle, Michael

Nosal, Vladimir

Odier, Céline

Oferrall, Erin
Omar, Abdelsimar

Pal, Pramod

Pan, Edward

Pandey, Sachin

Panisset, M.

Paquette, Caroline

Parks, Natalie

Parney, Ian

Parrent, Andrew

Pascarella, Angelo

Pasco, Paul Matthew

Pasternak, Stephen

Patel, Rakesh

Patel, Samir

Paton, Gillian

Peeling, Lissa

Pelz, David

Peng, Philip

Perez, Julio

Pfeffer, Gerald

Phillips, Stephen

Picillo, Marina

Pickett, Gwynedd

Pikula, Aleksandra

Pitz, Marshall

Plow, Ela

Poppe, Alexandre

Poulin, Stéphane

Pringle, Elizabeth

Prior, Peter

Prud'Homme, Michel

Pulcine, Liza

Purdy, Allan

Purohit, Abhishek

Rafay, Mubeen

Rajput, Alex

Ramaswamy, Vijay

Ramos, Ronald

Reebye, Rajiv

Rempel, Jeremy

Rheaume, Alan

Rigby, Heather

Rivest, Jean

Robinson, Larry

Rockwood, Kenneth

Rohani, Mohammad

Rosa-Neto, Pedro

Ross, Douglas

Ruiz-Lopez, Martha

Rumney, Peter

Rutka, James

Ryan, Monique

Sadeghian, Hamid

Sadikot, Abbas

Sadler, Mark

Sahjpaul, Ramesh

Saini, Jitender

Salehi, Fateme

Sallam, Yasmine

Samdup, Dawa
Sandoe, Claire

Saranza, Gerard

Sarma, Dipanka

Sarnat, Harvey

Satkunam, Lalith

Schellenberg, Kerri

Schipper, Hyman

Schmidt, Brian

Schurman, Lesley

Schutz, Peter

Selby, Kathryn

Serletis, Demitre

Shamy, Michel

Shankar, Jai

Sharma, Michael

Sharma, Sunjay

Sharma, Vikas

Sharp, Madeleine

Shetty, Aakash

Shoesmith, Christen

Shuaib, Ashfaq

Siddiqi, Ferhan

Siddiqi, Zaeem

Sim, Valerie

Sinclair, John

Skrobik, Yoanna

Slaoui, Tarik

Slavin, K

Slow, Elizabeth

Soh, Derrick

Soman, Teesta

Son, Maksim

Spacey, Sian

Sposato, Luciano

Starreveld, Yves

Steven, David

Stotts, Grant

Suchowersky, Oksana

Suller-Marti, Ana

Sultan, Darvesh

Sundaram, Arun

Taccone, Michael

Tampieri, Donatella

Tang-Wai, David

Taranath, Ajay

Tarnopolsky, Mark

Tartaglia, Maria Carmela

Tateno, Toru

Taylor, Sean

Teitelbaum, Jeanne

Teixeira Marques, Paula

Tellez-Zenteno, J.F.

ten Hove, Martin

Tetreault, Martine

Tibussek, Daniel

Tomek, Ales

Traboulsee, Anthony

Tsang, Derek

Tsivgoulis, G

Tso, Michael 
Tymchak, Zane Tyndel, Felix

Uchino, Ken

Udow, Sean

Valiante, Taufik

van Gerpen, Jay

van Landeghem, Frank

Veilleux, Martin

Venkat Raghavan,

Lakshmikumar

Vitali, Alexander
Vodopivec, Ivana

Wade, Kevin

Wagle Shukla, Aparna

Walker, Lisa

Walling, Simon

Wang, Gang

Wang, Shelly

Warman Chardon, Jodi

Wein, Francine

Wein, Theodore

Weise, Lutz
Wennberg, Richard

Wheatley, Matt

White, Chris

Winston, Paul

Wirrell, Elaine

Wolf, Amparo

Wong, John

Xia, Chenjie

Yamashita, Koji

Yang, Christine

Yeh, Steven
Yip, Stephen

Young, G. Bryan

Yu, Amy

Zacharia, Andre

Zerna, Charlotte

Zhou, Lily

Ziai, WC

Zinman, Lorne

Zochodne, Douglas 